\title{
Joining of Silver Nanowires by Femtosecond Laser Irradiation Method
}

\author{
Su Ding, Yanhong Tian*, Zhi Jiang and Chunqing Wang \\ State Key Laboratory of Advanced Welding and Joining, Harbin Institute of Technology, Harbin 150001, China
}

The silver nanowires with diameter around $100 \mathrm{~nm}$ and length up to $10 \mu \mathrm{m}$ were successfully synthesized by a simple polyol method. After the irradiation of fs laser, the silver nanowires melted at special spots because of the surface plasmon effect, which provide possible strategy for nanojoining. Silver nanowires could be joined together at the contact point as a result of the antenna effect with slight deformation. When the gap between two silver nanowires is larger, the self-generated nanoparticles in the middle of silver nanowires performed as medium to form nanojoints. [doi:10.2320/matertrans.MI201405]

(Received December 2, 2014; Accepted January 7, 2015; Published February 20, 2015)

Keywords: silver nanowires, nanojoining, femtosecond laser

\section{Introduction}

With the rapid development of nanotechnology, nanojoining becomes a difficult crossed threshold on the way of nanomaterials research. There are several methods have been reported, such as joule heating by electric, ${ }^{1)}$ electron or ion beam welding, ${ }^{2)}$ cold welding, ${ }^{3)}$ ultrasonic nanowelding, ${ }^{4)}$ femtosecond laser induced joining, ${ }^{5)}$ and soldering. ${ }^{6}$ ) However, methods operated in microscopy greatly limit the industrial application and soldering inquires a strict structure of nanowires. Fs laser irradiation method is a fast and convenient technology operated in atmosphere among these methods.

Zhou's group has done lots of work on the reaction between fs laser and micro- and nano-materials including engineering and welding. ${ }^{5,7,8)}$ Gold and silver nanoparticles have been reported to be welded by the fs laser induced method. However, it is still lack of experimental data and basic theory of the joining process. Here, we report the nanojoining process of silver nanowires under the fs laser irradiation which provides potential application in integration of nanodevices.

\section{Experimental Procedure}

Silver nanowires have been fabricated by a polyol method, employing polyvinylpyrrolidone (PVP) as surfactant and ethylene glycol (EG) as reduction. A small amount of ferrum nitrate $\left(\mathrm{Fe}\left(\mathrm{NO}_{3}\right)_{3}\right)$ was added to adjust the shape of products. The precipitate was collected by centrifugal separation and washed several times.

The silver nanowires solution was dispersed on glass to be irradiated by the fs laser. In our experiments, a Tisapphire laser system was operated to generate $40 \mathrm{fs}$ laser pulses at a repetition rate of $1 \mathrm{kHz}$ and $800 \mathrm{~nm}$ with Gaussian beam. A mechanical shutter was used to select the number of pulses per illumination. Morphology and structure of the nanowires and the nanojoints were checked with a field-emission scanning electron microscopy (SEM, FEI Quanta 200F) and a transmission electron microscopy (TEM, FEI Tecnai G2 F30) microscope operated at $300 \mathrm{kV}$.

*Corresponding author, E-mail: tianyh@hit.edu.cn

\section{Results and Discussions}

To synthesize the $\mathrm{Ag}$ nanowires, $0.8 \mathrm{mmol}$ silver nitrate, $1 \mathrm{mmol}$ PVP and $2.2 \mu \mathrm{mol} \mathrm{Fe}\left(\mathrm{NO}_{3}\right)_{3}$ were dispersed in a flask heating in an oil bath at $160^{\circ} \mathrm{C}$ for $1 \mathrm{~h}$. Figure 1 shows SEM and TEM images of the Ag nanowires. The nanowires have smooth surfaces with diameters around $100 \mathrm{~nm}$ and lengths up to $10 \mu \mathrm{m}$. For keeping a long time in alcohol the nanowires began to connect at the ends without PVP attached to the surface as indicated in Fig. 1(b). ${ }^{9)}$ The synthesis method followed former work and the structure of the silver nanowires was a five twinned structure as in papers. ${ }^{10)}$ The growth direction of silver nanowires is along [110] according to the high resolution TEM (HRTEM) image and the corresponding fast Fourier transformation (FFT) pattern as shown in Fig. 1(c). The SAED pattern in Fig. 1(d) ensured that the obtained nanowires are silver nanowires with pentatwinned structure that the two sets of spots indexed in the figure clearly show the twinned structure and the presence of a sequence of diffraction spots marked by arrows indicates the five-fold structure.

The as-prepared silver nanowires were dispersed on small pieces of glass as shown in Fig. 2(a). The glasses were placed under the irradiation of fs laser. The fs laser was focused by a lens with focal length of $30 \mathrm{~cm}$ and the total energy is $1.5 \mathrm{~W}$. In this paper, we use the fs laser with energy at $1.5 \mathrm{~W}$ and duration of $2 \mathrm{~ms}$. Figure 2(b) shows a typical morphology of the welded silver nanowires after the fs laser irradiation. There are nanopartiles at both ends of the silver nanowires and the two nanowires are joined together at the contact point with slight deformation. The TEM image as shown in Fig. 2(c) indicates that the two silver nanowires were fused together. The orientation of the joint area was different from both silver nanowires that there are boundaries between the joint area and the nanowires as marked in the image. This demonstrated the contact point of the two silver nanowires was melted and recrystallized under the effect of the fs laser. The pulse of the laser is very fast even quicker than the thermal transmission. The laser energy was local limited at the special spots including the ends and the contact points of silver nanowires. It can be seen in other segment of the silver nanowires maintained the original five twined structure. The fs laser works on special spots of the silver nanowire and other points are free of thermal shock, which is caused by the 

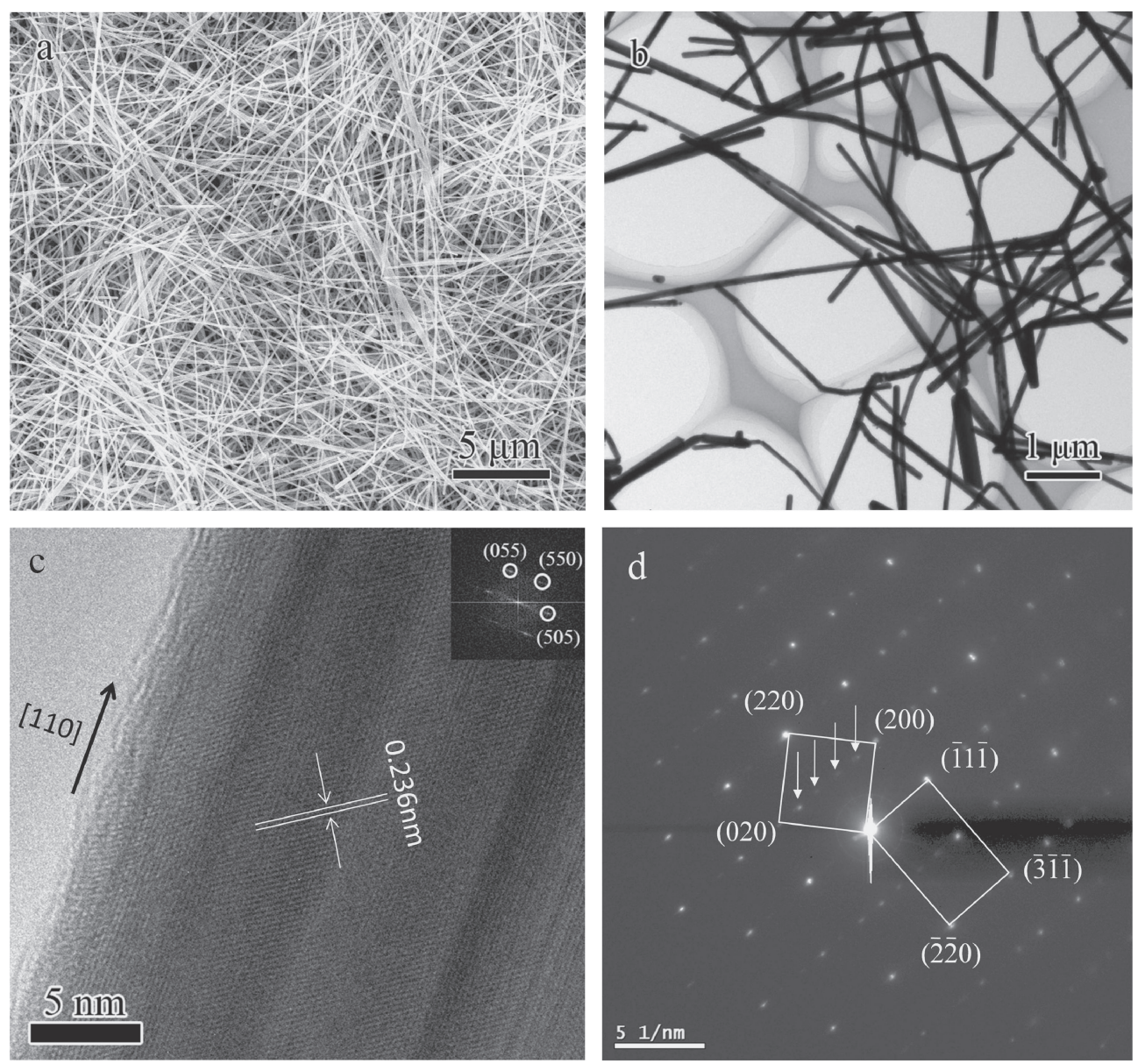

Fig. 1 (a) SEM and (b) TEM of the as-prepared silver nanowires. (c) HRTEM of silver nanowire, insert is the corresponding FFT transformation indicating the growth direction of the silver nanowire is along [110] as shown by the arrow; (d) SAED pattern of one silver nanowire.
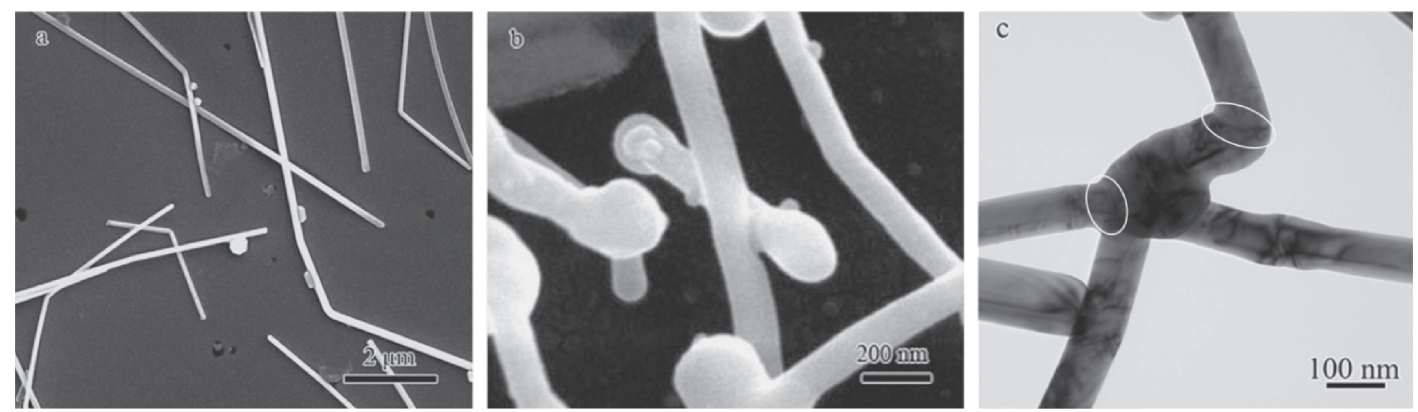

Fig. 2 (a) Silver nanowires before and (b) after the laser irradiation; (c) TEM image of the nanojoint.

surface plasmon response (SPR) of silver nanowires. When the silver nanowires were irradiated by laser, the energy was intense at the ends of silver nanowires which was also reported in pervious paper. ${ }^{5)}$ In addition, when the silver nanowire overlapped another one, it worked as an antenna leading energy into the nanowire at the bottom which made melting happen at the contact point. ${ }^{11,12}$ However, the joining did not happen at every contact point. When the distance between nanowires is large, the energy is not big enough for joining. However, a possible joining method for that condition was observed in our experiment.

To introduce the joining strategy of two nanowires with larger gaps between them, the responses of the silver nanowires to the fs laser would be given first. The polarized orientation of the fs laser significantly influenced the energy distribution around the silver nanowires. In our experiments, the silver nanowires were dispersed on the glasses randomly. The angle between the fs laser and the silver nanowires is various. When the laser is perpendicular to the silver naonowires, the energy is concentrated at the ends. When the incident laser is parallel with the silver nanowires, the energy distribution is harmonic vibration as the laser. ${ }^{5)}$ As shown in Fig. 3(a), there are several particles generating at the surface of one silver nanowire due to the uneven distribution of energy. In this condition, maybe the direction of the laser is along the growth orientation of the silver 

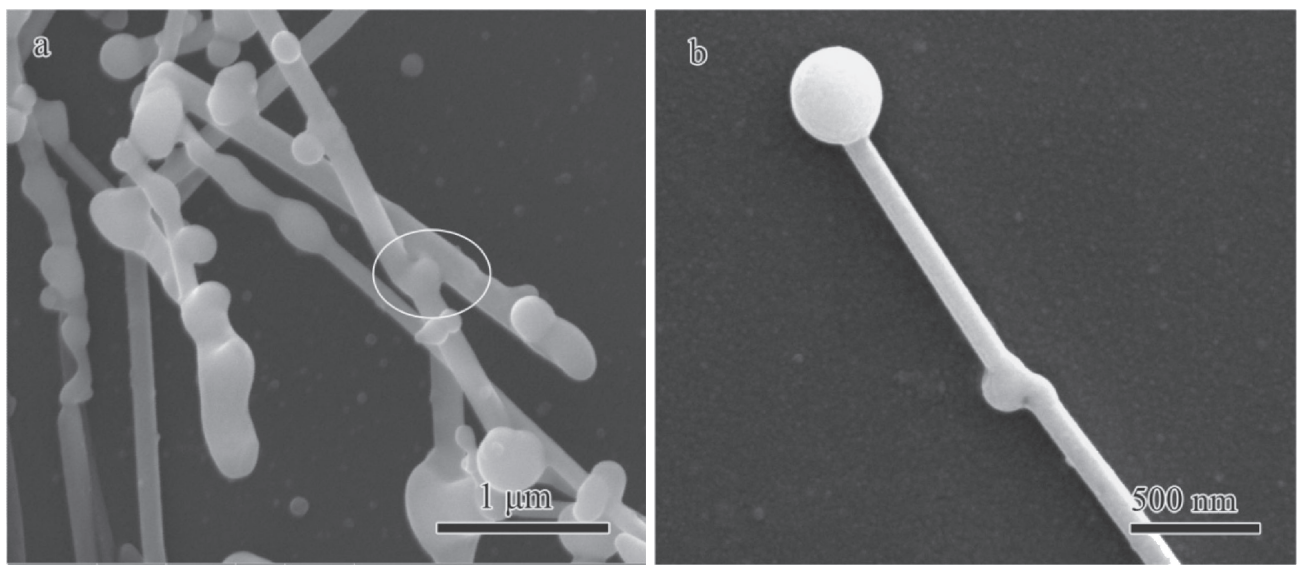

Fig. 3 (a) Nanoparticles generated on the surface of silver nanowires, nanojoint formed as marked by the circle; (b) a single nanowire with deformation caused by the melting.

nanowires. However, not all the silver nanowires melted to generate nanoparticles. The diameter of the nanowires was declined indicating the nanoparticles at the surface were self generated consuming the silver nanowires. These silver nanowires were much shorter than the original nanowires possibly due to the fracture during the production of the nanoprarticles. Some nanoparticles separated from the silver nanowires. The nanoparticles provided a possible method to join silver nanowires as marked by the circle in the SEM image. The newly generated nanoparticles performed as medium to join the two silver nanowires. The nanoparticle generated at one nanowire and connected to another one during the melting and recrystallized process. The distance between the two silver nanowires was larger to hold a newly generated nanoparticle between them. The nanoparticles generated at the hot spots where energy adsorption was enhanced. During the growing up of the parctiles, the nanoparticle easily adhered to another silver nanowire to reduce the surface energy. As a result, the nanowire deformed during the process. A similar structure was illustrated in Fig. 3(b). There were two nanoparticles in the silver nanowire, which was at the end and the other was in the middle. It seemed like two nanowires with a small particle between them. However, the diameters of the nanowire at two sides of the nanopartice were nearly the same demonstrating the two segments belonged to one nanowire. The deformation occurred during the melting process. The selfgenerated nanoparticles could join nanowires together as a medium.

\section{Conclusion}

Silver nanowires were successfully synthesized by a simple polyol method. The as-prepared silver nanowires were five tiwned structure with diameter around $100 \mathrm{~nm}$ and length up to $100 \mu \mathrm{m}$ through the characterization by SEM and TEM. A fs laser irradiation technology was demonstrated to be an effective method to join silver nanowires. As a typical plasmon, the silver nanowires melted at specific spots. The ends of silver nanowires formed nanoparticles. At the contact points, the overlapping nanowire performed as antenna leading energy to the bottom nanowire. This antenna effect caused the joining happen by melting at the contact spots. The nanoparticles generated in the middle of the silver nanowires also provided a way to form nanojoints. The selfgenerated nanoparticles performed as medium. Our study shows a new view of nanojoining which plays a significant role in nanodevices integration. However, there is still a lot of work to do to clearly describe the effect of fs laser to the silver nanowires and precisely join silver nanowires as a desired configuration.

\section{Acknowledgments}

This work has been supported by Program for New Century Excellent Talents in University (NCET-13-0175).

\section{REFERENCES}

1) H. Tohmyoh, T. Imaizumi, H. Hayashi and M. Saka: Scr. Mater. 57 (2007) 953-956.

2) S. Xu, M. Tian, J. Wang, J. Xu, J. M. Redwing and M. H. W. Chan: Small 1 (2005) 1221-1229.

3) Y. Lu, J. Y. Huang, C. Wang, S. Sun and J. Lou: Nat. Nanotechnol. 5 (2010) 218-224.

4) C. Chen, L. Yan, E. Kong and Y. Zhang: Nanotechnol. 17 (2006) $2192-$ 2197.

5) L. Liu, P. Peng, A. Hu, G. Zou, W. Duley and Y. Zhou: Appl. Phys. Lett. 102 (2013) 073107.

6) Y. Peng, T. Cullis and B. Inkson: Nano Lett. 9 (2009) 91-96.

7) A. Hu, P. Peng and H. Alarifi: J. Laser Appl. 24 (2012) 042001.

8) A. Hu, Y. Zhou and W. W. Duley: Open Surf. Sci. J. 3 (2011) 42-49.

9) P. Peng, L. Liu, A. P. Gerlich, A. Hu and Y. Zhou: Part. Part. Syst. Char. 30 (2013) 420-426.

10) B. Wiley, Y. Sun and Y. Xia: Langmuir 21 (2005) 8077-8080.

11) E. Garnett, W. Cai, J. Cha, F. Mahmood, S. Connor, M. Christoforo, Y. Cui, M. McGehee and M. Brongersma: Nat. Mater. 11 (2012) 241-249.

12) P. Ghenuche, S. Cherukulappurath, T. Taminiau, N. Hulst and R. Quidant: Phys. Rev. Lett. 101 (2008) 116805. 